served after Adv/HSV1-TK treatment alone. This finding indicates that the chronic inflammation may be mediated by damage induced by the 'bystander effect' in concert with immunity directed against adenoviral proteins.

One of the most intriguing findings of Dewey et al. is that localized demyelination was found in the area where the tumor had originally resided. There are several mechanisms by which this demyelination may have occurred. First, peritumoral edema induced during tumor expansion could have compacted surrounding normal tissue, leading to oligodendrocyte death and localized demyelination. Alternatively, products released by activated macrophages (such as nitric oxide or superoxide) and/or $\mathrm{CD}^{+} \mathrm{T}$ cells (such as perforin or granzyme B) may lead to the nonspecific destruction of oligiodendrocytes and/or myelin. It is unlikely that the observed demyelination is autoimmunemediated, as the area of demyelination was restricted to the original tumor bed, whereas autoimmune demyelination might be expected to be present with a much wider distribution. Yet this important possibility cannot be ignored. The presence of an immunospecific inflammatory reaction against tumor antigens could conceivably lead to the generation of a response against unintended native antigens through the putative mechanism of epitope spreading $^{6,7}$. Such a mechanism would represent a considerable 'downside' to suicide gene therapy may exist.

This study raises important points regarding current adenoviral-based clinical trials for human gliomas. There should be concern about the potential of this technique to induce chronic active inflammation which may ultimately have negative consequences on the functional integrity of the brain parenchyma. It will be important to determine whether these findings extend from the animal model to human brain tumors. Further study to identify the antigenic stimulus of this chronic inflammation is warranted to ascertain whether it will interfere with the administration of additional vector or the induction of a secondary immune re- sponse upon tumor recurrence.

1. Freeman, S.M., Ramesh, R. \& Marrogi, A.J. Immune system in suicide-gene therapy. Lancet $349,2-3$ (1997).

2. Dewey, R.A. et al. Chronic brain inflammation and persistent herpes simplex virus 1 thymidine kinase expression in survivors of syngeneic glioma treated by adenovirus-mediated gene therapy: Implications for clinical trials. Nature Med. 5, 1256-1263 (1999).

3. Kruse, C.A. et al. A rat glioma model, CNS-1, with invasive characteristics similar to those of human gliomas: A comparison to 9L gliosarcoma. J. NeuroOncol. 22, 191-200 (1994).

4. Yang, Y. et al. Cellular immunity to viral antigens limits E1-deleted adenoviruses for gene therapy. Proc Natl. Acad. Sci. USA 91, 4407-4411 (1994).

5. Worgall, S. et al. Selective expansion of alveolar macrophages in vivo by adenovirus-mediated transfer of the murine granulocyte-macrophage colonystimulating factor CDNA. Blood 93, 655-666 (1999).

6. McRae, B.L., Vanderlugt, C.L., Dal Canto, M.C., \& Miller, S.D. Functional evidence for epitope spreading in the relapsing pathology of experimental autoimmune encephalomyelitis. J. Exp. Med. 182, 75-85 (1995).

7. Miller, S.D. et al. Evolution of the T-cell repertoire during the course of experimental immune-mediated demyelinating diseases. Immunol. Rev. 144, 225-244 (1995).

Department of Pathology

Dartmouth Medical School

Dartmouth Hitchcock Medical Center,

Lebanon, New Hampshire 03756, USA

\title{
Shedding new light on luminance perception
}

One of the oldest puzzles in vision research involves the mechanisms by which the context of a stimulus can influence the perception of its relative brightness. For example, the two surfaces of the object shown here are physically identical (of equal luminance), but appear to be different, because of opposing light and dark luminance gradients along a step boundary. These different luminance gradients make the upper square appear darker than the lower square. Vision researchers have long thought that this phenomenon, known as the 'Cornsweet effect', could be explained by the receptive field properties of retinal ganglion cells.

Dale Purves and colleagues have re-examined this hypothesis, and in the 1 October issue of The Journal of Neuroscience, they propose that perceptions of luminance are not determined by retinal physiology, but by empirical associations made in the circuitry of the visual cortex. Investigation of different factors that enhance or re- duce the Cornsweet effect suggest that the brain accumulates information from

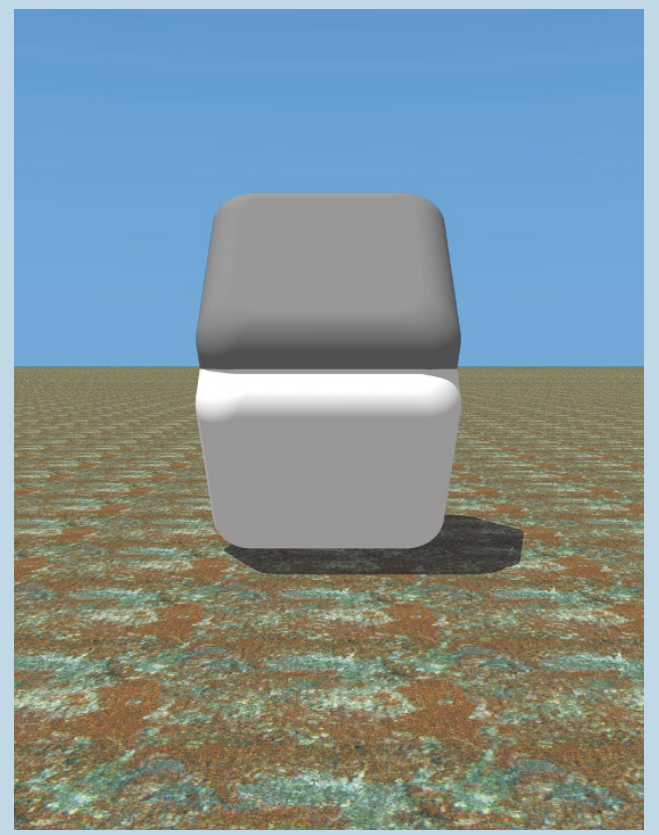

past visual stimuli and applies it to the stimulus confronting the observer at any given moment. Thus, the various pieces of information included in this image, such as the luminance gradient, perspective, orientation, texture and background, all indicate to the viewer, based on past visual experience, that the two areas are likely to differ in brightness. Accordingly, this is the perception experienced.

The authors of the paper believe that this empirical strategy of visual processing has been selected for throughout evolution. "Since all visual stimuli are ambiguous, the most efficient way for an animal to respond is to generate an association based on what the stimulus has usually turned out to be, and then act upon that," explained Purves. "The animal that couldn't do this effectively probably got eaten by a sabertoothed tiger."

Purves and colleagues are also investigating this empirical theory in terms of other visual qualities that affect luminance perception, such as color (reported in the November issue of Nature Neuroscience).

KRISTINE NovaK 